\title{
A Novel Technique to Prevent the Sinus Membrane Collapse for Maxillary Sinus Floor Augmentation without Bone Graft: Technical Note
}

Antonio Scarano ${ }^{1}$, M.D. D.D.S, Francesco Carinci², Felice Lorusso ${ }^{3}$, Francesco Inchingolo ${ }^{4}$.

${ }^{1}$ Professor of Oral Surgery, Department of Medical, Oral and Biotechnological Sciences and ${ }^{2}$ Center for Research on Ageing and Translational Medicine (CeSI-MeT), University of Chieti-Pescara, Italy; ${ }^{2}$ Chief of Maxillofacial Surgery, University of Ferrara, Italy

${ }^{3}$ Department of Medical, Oral and Biotechnological Sciences, University of Chieti-Pescara, Italy. ${ }^{4}$ Department of Interdisciplinary Medicine, University of Bari “Aldo Moro”, Bari, Italy Corresponding author: Antonio Scarano, M.D., D.D.S.; Via dei Vestini 31; 66100 Chieti; (Italy); Fax: +39-0871-3554099; E-mail:ascarano@unich.it

\begin{abstract}
A variety of surgical techniques have been developed to reconstruct the posterior maxilla when bone volume is insufficient. A barrier membrane or bone window pushed inside the sinus cavity as the "roof" of the sinus cavity for preserve the space and help bone. The heterologous cortical lamina is used for the mechanical support, without any grafting material, of sinus membranes resulting in only bone tissue formation and not mixed with the graft.
\end{abstract}

Key words: bone graft, resorbable barrier, sinus lift, heterologous cortical lamina.

Running title: Sinus lift with a rigid barrier 


\section{Introduction}

Rehabilitation of the edentulous posterior maxilla with dental implants may be a problem because of insufficient bone volume produced by buccolingual and/or apic occlusal atrophy of the edentulous alveolar crestal bone and pneumatization of the maxillary sinus. In this anatomical situation, it can be very difficult to obtain primary stability because of the absence of a useful quantity of cortical bone and for the loose structure of type IV spongious bone which prevents an implant migration into the sinus[1,2].

The grafting materials that are utilized in floor augmentation could provide for a bone formation process, by replacing the bone materials, due to capillary infiltration, and support of the implants[3]. These grafting materials help to maintain space between the basal bone and membrane.

A new bone formation is achieved in the sinus after bone grafting it is possible to implant placement. However the possibility of new bone formation with a membrane elevation without bone graft in the maxillary sinus has been reported in human studies[4,5] Spontaneous bone formation inside the maxillary sinus has been noted after removal of a cyst[6], removal displaced implant[7] and a extraction socket[8]. The sinus membrane elevation technique was investigated in patients referred for sinus augmentation[4,9]. Sinus membrane stabilization is a requirement for bone formation. It must be pointed out that maxillary sinus pneumatization could be the result of positive intrasinus air pressure caused by respiration, and this pressure might promote resorption and new pneumatization after maxillary sinus augmentation. To avoid this Borges et al.[9] pushed the lateral bone window inside the sinus cavity, using this thin bone as the "roof" of the secluded cavity. The limitation of this technique is the distance between the antrostomy and the nose wall, in fact, in the case of a large distance the bone window is insufficient for supporting the sinus membranes.

To avoid the sinus membrane collapse for maxillary sinus floor augmentation without bone graft, a novel technique for placing a bone lamina (lamina, Tecnoss, Coazze, Italy) in antrostomy is presented. 
This technique uses a cortical bone lamina of $1 \mathrm{~mm}$ thickness, modelled and positioned in the sinus as a new sinus. This membrane is rigid and stability improves through the two lines from $2-3 \mathrm{~mm}$, mesial and distal created at the top of the antrostomy. After elevation of the Schneiderian membrane was accomplished, two lines of 2-3 mm, mesial and distal, were created at the top of the antrostomy (Fig.12). The antrostomy was measured with a periodontal probe for cutting the membrane to size.

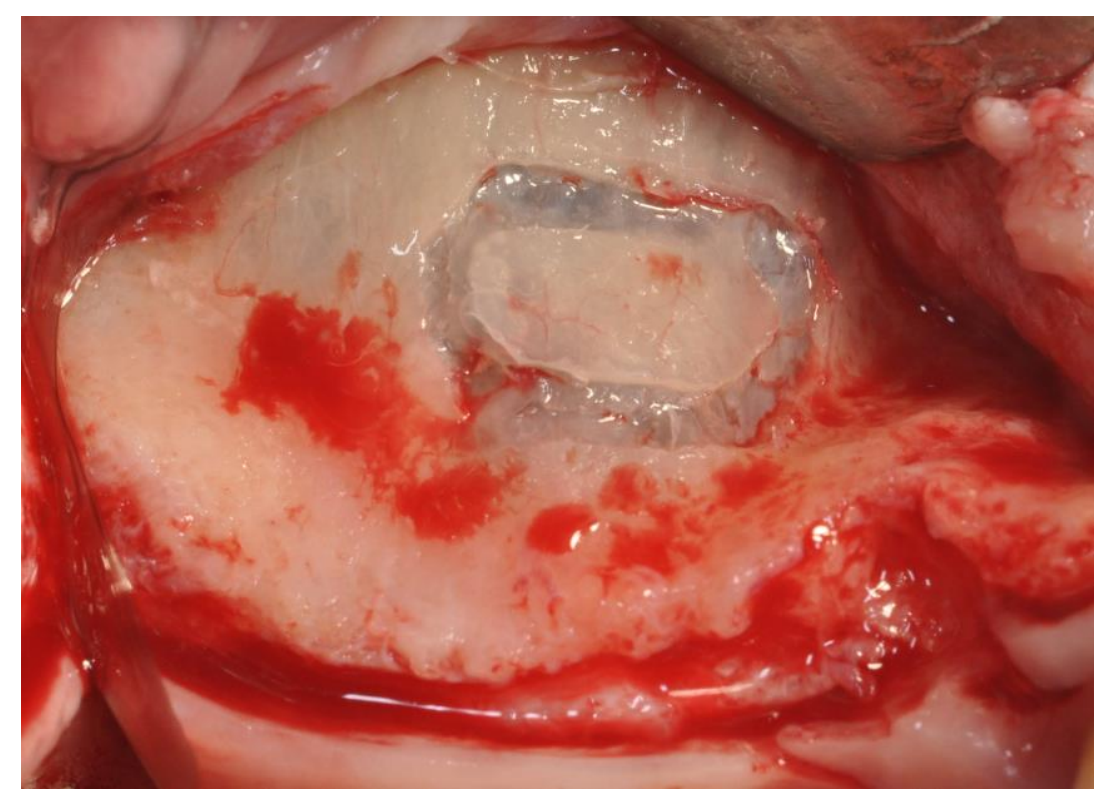

1) The antrostomy is performed and removed. 


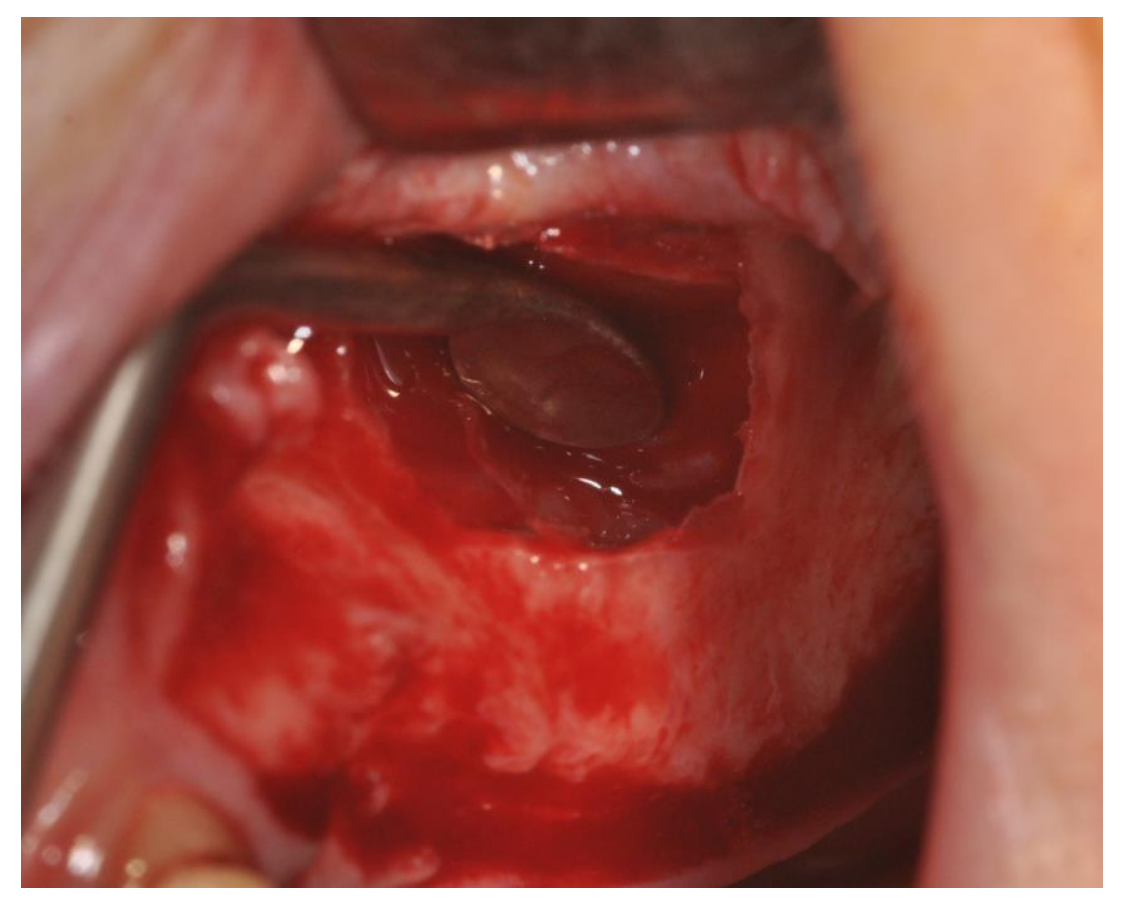

2) Schneiderian membrane is mobilizing.

This heterologous barrier is washed with saline solution for $10 \mathrm{~min}$. After water contact the bone lamina softens and it is possible to shape it allowing to fit the anatomic curvature of the sinus. The cortical laminar membrane is first softened and then imbibed with the patient's blood for 2-3 minutes. The half heterologous membrane was positioned on these lines and pushed to the nose wall of the sinus (Fig. 3), when the coagulum is observed underneath the elevated sinus mucosa the other half was folded to cover the window (figs. 4-6). This procedure was performed carefully to avoid tearing or folding of the bone lamina. 


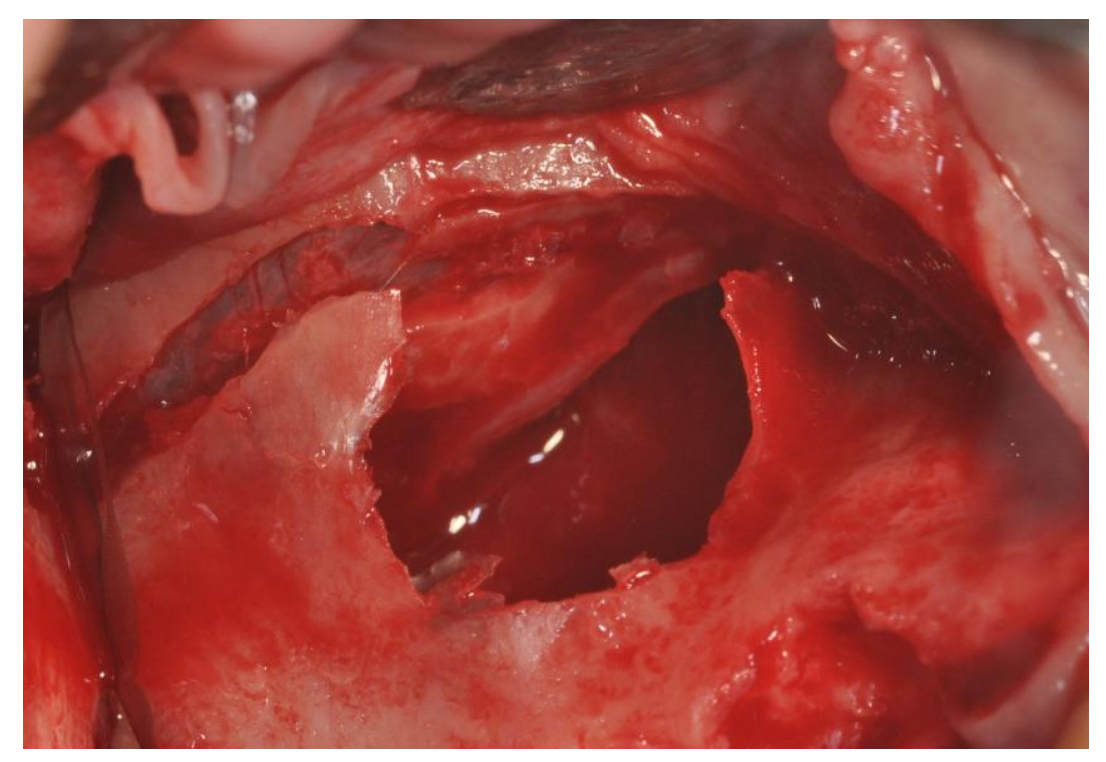

3. Schneiderian membrane is mobilizing and two lines of 2-3 $\mathrm{mm}$, mesial and distal, is created at the top of the antrostomy.

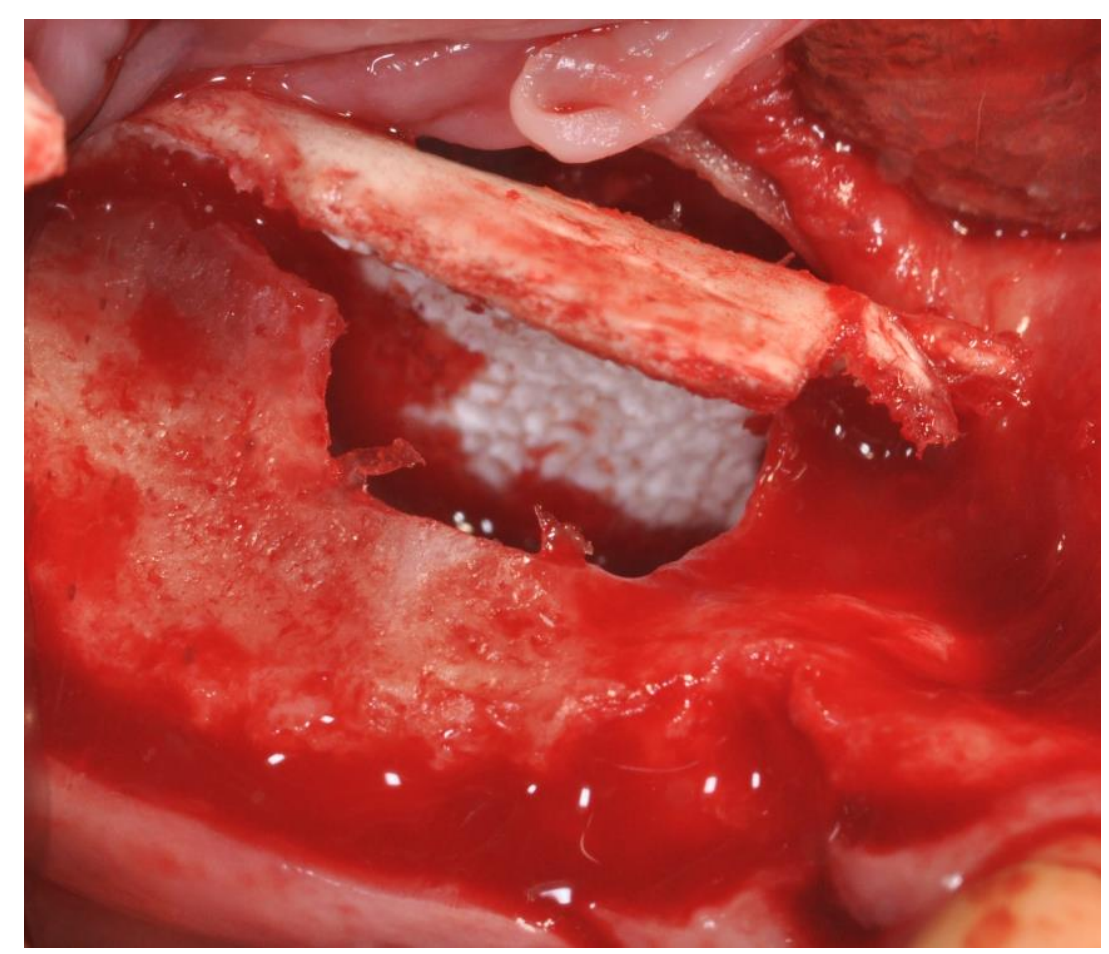

4. The half heterologous membrane is positioned on lines and pushed to the nose wall of the sinus. 


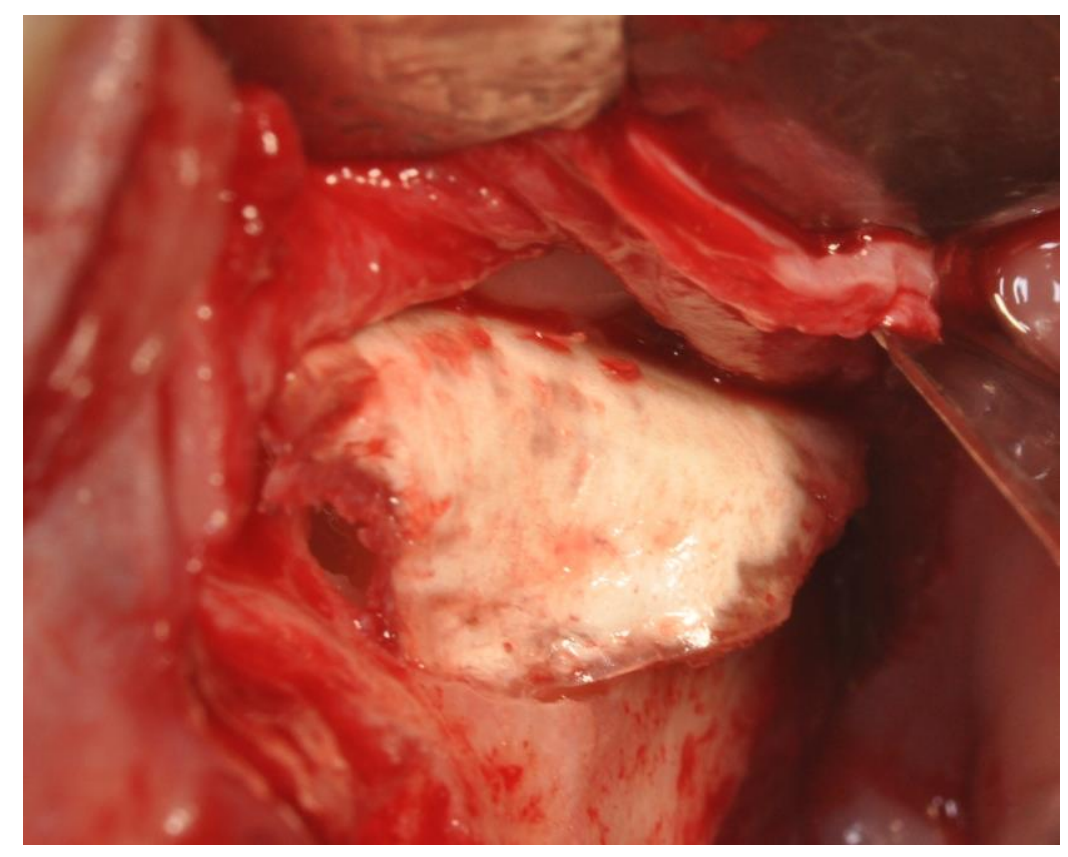

5. The other half is folded to cover the window.

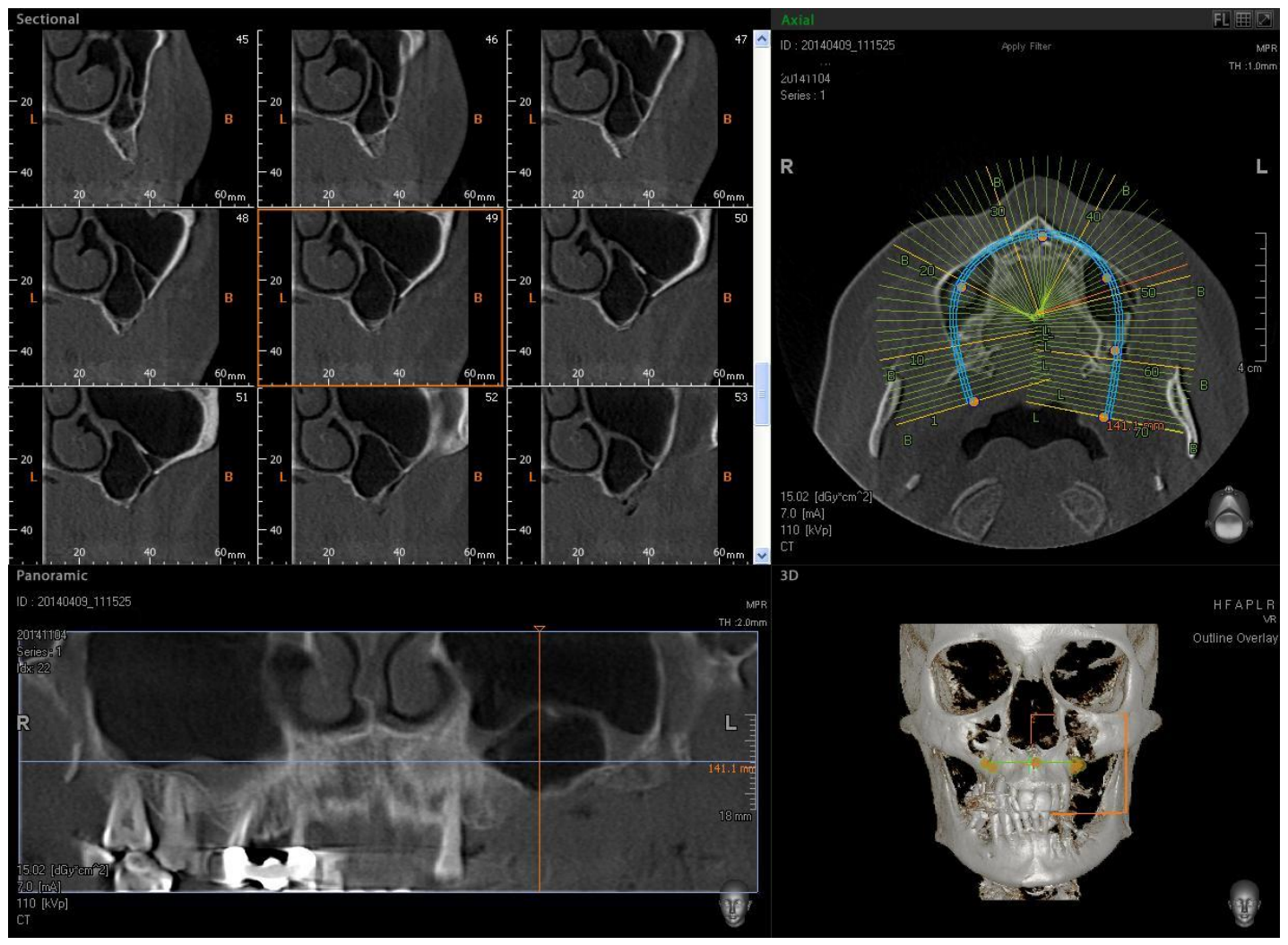

6. CBCT after sinus lifting with bone lamina for preserve the space. 
The heterologous cortical lamina is a valid technique for mechanical support of the sinus membrane for help only bone tissue formation and not a mix with bone graft. It is the experience of the authors that this procedure prevents sinus membrane collapse.

\section{REFERENCES}

1. Scarano, A.; Perrotti, V.; Carinci, F.; Shibli, J. A. Removal of a migrated dental implant from the maxillary sinus after 7 years: a case report. Oral Maxillofac Surg 2011, 15, 239-243, doi:10.1007/s10006-010-0243-8.

2. $\quad$ Fürst, G.; Gruber, R.; Tangl, S.; Zechner, W.; Haas, R.; Mailath, G.; Sanroman, F.; Watzek, G. Sinus grafting with autogenous platelet-rich plasma and bovine hydroxyapatite. A histomorphometric study in minipigs. Clin Oral Implants Res 2003, 14, 500-508.

3. Froum, S. J.; Tarnow, D. P.; Wallace, S. S.; Rohrer, M. D.; Cho, S. C. Sinus floor elevation using anorganic bovine bone matrix (OsteoGraf/N) with and without autogenous bone: a clinical, histologic, radiographic, and histomorphometric analysis--Part 2 of an ongoing prospective study. Int J Periodontics Restorative Dent 1998, 18, 528-543.

4. Lundgren, S.; Andersson, S.; Gualini, F.; Sennerby, L. Bone reformation with sinus membrane elevation: a new surgical technique for maxillary sinus floor augmentation. Clin Implant Dent Relat Res 2004, 6, 165-173.

5. Felice, P.; Scarano, A.; Pistilli, R.; Checchi, L.; Piattelli, M.; Pellegrino, G.; Esposito, M. A comparison of two techniques to augment maxillary sinuses using the lateral window approach: rigid synthetic resorbable barriers versus anorganic bovine bone. Five-month post-loading clinical and histological results of a pilot randomised controlled clinical trial. Eur J Oral Implantol 2009, 2, 293306. 
6. Lundgren, S.; Andersson, S.; Sennerby, L. Spontaneous bone formation in the maxillary sinus after removal of a cyst: coincidence or consequence? Clin Implant Dent Relat Res 2003, 5, 78-81.

7. Scarano, A.; Piattelli, A.; Iezzi, G.; Varvara, G. Spontaneous bone formation on the maxillary sinus floor in association with surgery to remove a migrated dental implant: a case report. Minerva Stomatol 2014, 63, 351-359.

8. Jung, Y.-S.; Chung, S.-W.; Nam, W.; Cho, I.-H.; Cha, I.-H.; Park, H.-S. Spontaneous bone formation on the maxillary sinus floor in association with an extraction socket. Int J Oral Maxillofac Surg 2007, 36, 656-657, doi:10.1016/j.ijom.2007.01.013.

9. $\quad$ Borges, F. L.; Dias, R. O.; Piattelli, A.; Onuma, T.; Gouveia Cardoso, L. A.; Salomão, M.; Scarano, A.; Ayub, E.; Shibli, J. A. Simultaneous sinus membrane elevation and dental implant placement without bone graft: a 6-month follow-up study. J. Periodontol. 2011, 82, 403-412, doi:10.1902/jop.2010.100343. 